\title{
CBP-mediated post-translational N-glycosylation of BRCA2
}

\author{
HABIBUR SIDDIQUE, VEENA N. RAO and E. SHYAM P. REDDY \\ Cancer Biology Program, Department of OB/GYN, Morehouse School of Medicine, Georgia Cancer Center \\ for Excellence, Grady Health System, 80 Jesse Hill Jr. Drive, Atlanta, GA 30303, USA
}

Received February 27, 2009; Accepted April 22, 2009

DOI: 10.3892/ijo_00000351

\begin{abstract}
CREB binding protein (CBP) is a transcriptional cofactor with intrinsic histone acetyl transferase activity (HAT). We have observed that CBP interacts with BRCA2 and mediates post-translational glycosylation of BRCA2. The binding of $\mathrm{CBP}$ to the amino-terminal region of BRCA2 is necessary for the glycosylation at residue 272 of BRCA2. Digestion with peptide $\mathrm{N}$-glycosidase $\mathrm{F}$ indicates that the glycosylation of BRCA2 is N-linked. It is possible that this novel CBP-mediated post-translational N-glycosylation activity alters the conformation of CBP-interacting proteins, leading to regulation of gene expression, cell growth and differentiation.
\end{abstract}

\section{Introduction}

The transcriptional cofactor CBP interacts with a number of cellular proteins, generally enhancing transcriptional function of its partners (1). CBP possesses intrinsic histone acetyl transferase activity and acetylates several CBP-interacting proteins, including p53, BRCA1, nuclear receptors, CREB, GATA-1, ELK-1, c-myb, E1A, and HIV-1 Tat $(2,3)$. Acetylation of p53 increases its DNA binding activity, presumably by altering the conformation of p53 (3). Previously, we reported an alternatively spliced isoform of BRCA2 (4). Since the aminoterminal region of $\mathrm{BRCA} 2$ is involved in transcriptional activation $(5,6)$, we tested whether BRCA2 interacts with CBP. A schematic representation of interacting and functional domains of CBP is shown in Fig. 1A. CBP1 (aa 461-662) binds to several transcriptional factors, including CREB, BRCA1, c-jun, c-myb, Tax, ATF-1, and YY1, whereas CBP2 (aa 1620-1877) interacts with E1A, SV40 large T, GATA-1, jun $\mathrm{B}$, Tat, and polyoma large $\mathrm{T}$ antigen $(2,3)$.

In eukaryotic cells, $\mathrm{N}$-glycosylation is an essential process that regulates protein folding, secretion, degradation and

Correspondence to: Professor E. Shyam P. Reddy, Cancer Biology Program, Department of OB/GYN, Morehouse School of Medicine, Georgia Cancer Center for Excellence, Atlanta, GA 30303, USA

E-mail: ereddy@msm.edu

Key words: BRCA2, CBP, N-glycosylation, cancer, protein degradation stability $(7,8)$. This process takes place cotranslationally during the translocation of nascent protein to the endoplastic reticulum. We report here, however, an exception to this process. We show that $\mathrm{CBP}$ binds to BRCA2 and mediates post-translational N-glycosylation of BRCA2. By use of deletions and point mutations of BRCA2, we have assigned $\mathrm{Asn}_{272}$ as the potential glycosylation site. Furthermore, we demonstrate that the HAT domain of CBP mediates this posttranslational $\mathrm{N}$-glycosylation. To our knowledge this is the first report that a transcriptional cofactor, like CBP, may mediate $\mathrm{N}$-linked glycosyltransferase activity.

\section{Materials and methods}

Plasmid construction and purification of proteins. Aminoterminal fragments of BRCA2 gene were generated by PCR and cloned in frame in pGEX-2T vectors (Pharmacia) to produce GST-fusion proteins in E. coli. GST-CBP constructs were a gift from Dr Tony Kouzarides. The constructs were trans-formed in E. coli BLR(DE3)pLysS competent cells (Novagen), and fusion proteins were purified. Similarly, the above BRCA2 cDNA fragments were cloned into pCDNA3 or pcDNA3/His for in vitro transcription and translation.

GST-pull down assay. PCDNA/BRCA2 DNAs were linearized with EcoRI that cleaved at aa 272. In vitro transcriptions were performed using a kit from Stratagene. Translated proteins were labeled with $\left[{ }^{35} \mathrm{~S}\right]$-methionine in a preparation of lysed rabbit reticulocytes (total volume, $25 \mu \mathrm{l}$ ) in the presence of Gst-CBP beads with occasional shaking. Where indicated, microsomal membranes were added (Promega). After $1 \mathrm{~h}$ at $30^{\circ} \mathrm{C}, 125 \mu \mathrm{l}$ of binding buffer [20 mM Tris- $\mathrm{HCl}(\mathrm{pH} 7.8)$, $150 \mathrm{mM} \mathrm{NaCl}, 2.5 \mathrm{mM} \mathrm{MgCl}_{2}, 0.1 \mathrm{mM}$ DTT and $0.05 \%$ $\mathrm{NP}-40$ ] was added and the preparation was rotated at $4^{\circ} \mathrm{C}$ for an additional $1 \mathrm{~h}$. The beads were washed in binding buffer and analyzed in SDS-PAGE. Bound proteins were visualized with either autoradiography or image analyzer (Fuji). PNGase, $\lambda$ PPase, and O-glycosylase treatments were performed according to manufacturer's instructions (Boehringer and Promega).

Immunoprecipitation and Western blots. MCF-7 cells were transfected with the PCDNA-HisC fusion construct of BRCA2 (aa 1-272) and PCDNA-CBP by use of Fugene 6 (Boehringer). After $72 \mathrm{~h}$, cells were harvested and lysed in buffer containing $50 \mathrm{mM}$ Tris- $\mathrm{HCl}, \mathrm{pH} 8.0 ; 150 \mathrm{mM} \mathrm{NaCl} ; 5$ mM EDTA; $0.5 \% \mathrm{NP}-40$ and protease inhibitor cocktail (Boehringer). 
A

Binding to BRCA-2
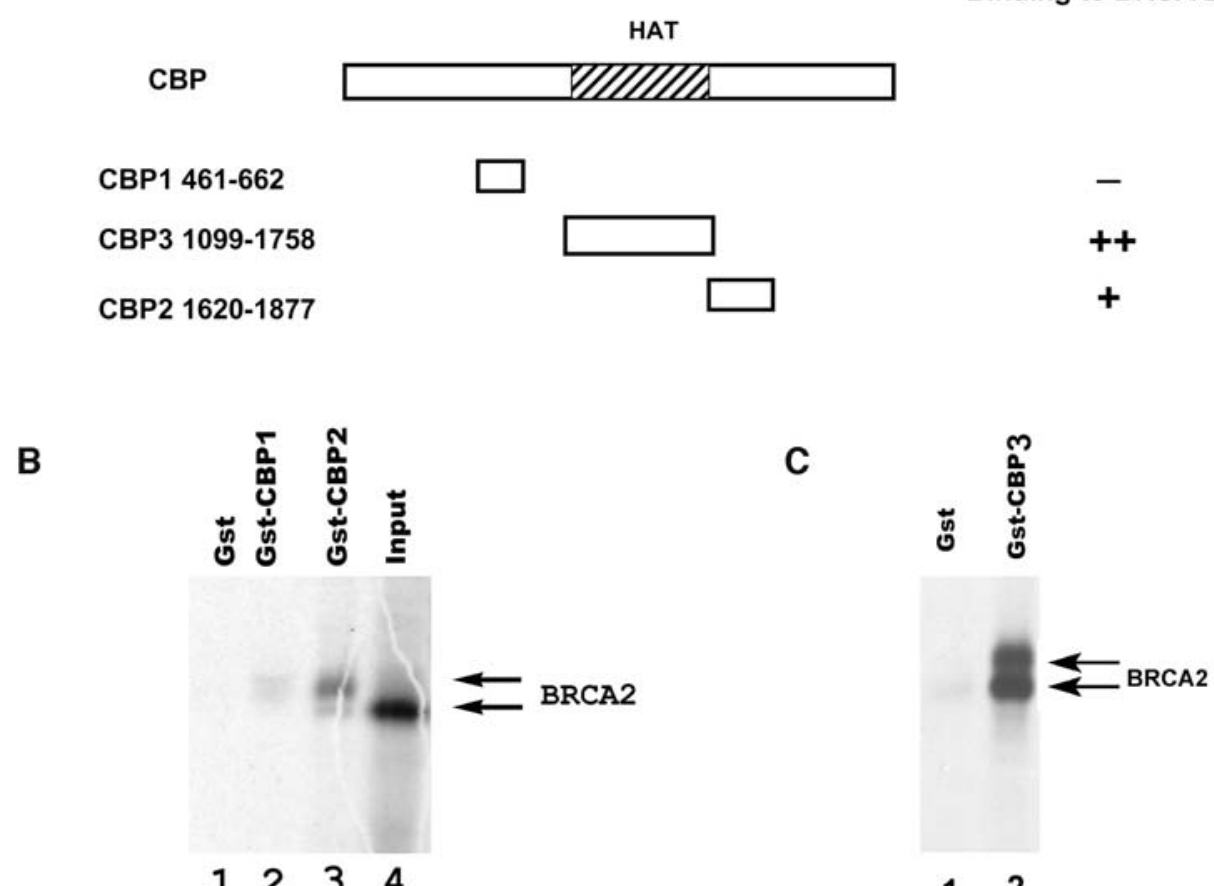

$\begin{array}{llll}1 & 2 & 3 & 4\end{array}$

12

Figure 1. (A), Schematic representation of domains of CBP used for interaction studies with BRCA2 (aa 1-272). (B), Interaction between CBP and BRCA2. In vitro translated BRCA2 (aa 1-272) was subjected to GST-pull down assay using GST-CBP1 (lane 2), GST-CBP2 (lane 3). Lane 1, GST control and lane 4, 5\% of input. (C), Binding and modification of BRCA2 by CBP3. In vitro translated BRCA2 (aa 1-272) was subjected to GST-pull down assay using GST-CBP3. Lane 1, GST and lane 2, GST-CBP3.

aa $1-272$

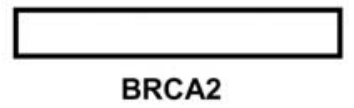

aa $1-188$

aa $188-272$
Posttranslational modification
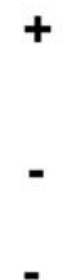

Figure 2. The binding of CBP to the amino-terminal region of BRCA2 is necessary for the post-translational modification of BRCA2.

After 20 min on ice, extracts were cleared by centrifugation. Extracts were incubated with CBP antibody (Santa Cruz) for $2 \mathrm{~h}$ at $4^{\circ} \mathrm{C}$. Protein A/G $(15 \mu \mathrm{l})$ plus agarose was added, and the preparation was rotated overnight. Beads were washed three times with protein binding buffer and subjected to electrophoresis. The gel was transferred to Hybond nitrocellulose paper and blotted with Xpress antibody (Invitrogen) by use of a Western blot kit (Amersham).

\section{Results}

Interaction of the HAT domain of $C B P$ with BRCA2. To test whether BRCA2 interacts with $\mathrm{CBP}$, we subjected the in vitro translated amino-terminal region of BRCA2 (aa 1-272) to GST-pull down assay using GST-CBP1 (aa 461-662) and GST-CBP2 (aa 1620-1877) (Fig. 1A). BRCA2 bound to CBP2 and resulted in a higher molecular weight product that was slower migrating, indicating post-translational modification (Fig. 1B, lane 3). Similarly, we tested the interaction of BRCA2 with the GST-CBP3 region (aa 1099-1758), which comprises the entire HAT of CBP. BRCA2 bound strongly to CBP3 and showed greater post-translational modification (Fig. 1C).

CBP-mediated post-translational modification of BRCA2. To determine which region of BRCA2 is responsible for posttranslational modification mediated by $\mathrm{CBP}$, we translated BRCA2 (aa 1-188) in vitro and subjected it to GST-pull down assay using GST-CBP3. Our results demonstrate that BRCA2 (1-188) bound to GST-CBP3, but was not modified posttranslationally, suggesting that aa 188-272 of the carboxyterminal region were the target of this modification (Fig. 2). 


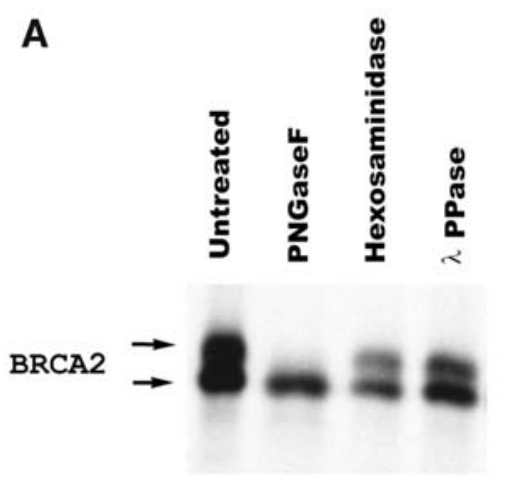

$\begin{array}{llll}1 & 2 & 3 & 4\end{array}$
B

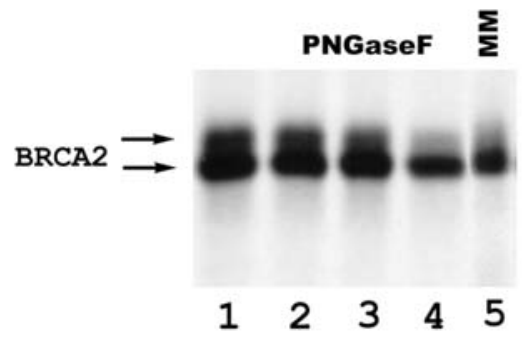

Figure 3. Characterization of post-translationally modified BRCA2 using enzymatic digestion. (A), In vitro translated BRCA2 (aa 1-272) was subjected to GST-CBP3 pull down assay. BRCA2 bound to GST-CBP3 was treated with various enzymes. Lane 1, no enzyme; lane 2, PNGase F; lane 3, $\lambda$ PPase; lane 4, O-glycosidase. (B), BRCA2 (aa 1-272) bound to GST-CBP3 was treated with increasing concentrations of PNGase F. Lane 1, no enzyme; lanes 2, 3, 4 indicate 1, 2 and 3 units of PNGase F respectively; lane 5, translation was carried out in the presence of $2 \mu 1$ of pancreatic microsomal membrane (Promega). The upper arrow and the lower arrow indicate glycosylated and non-glycosylated products of BRCA2 respectively.

In contrast, in vitro translated BRCA2 (aa 188-272) did not show appreciable binding to GST-CBP3 and also posttranslational modification. These results (Fig. 2) suggest that the binding of the amino-terminal region $(1-188)$ of BRCA2 to $\mathrm{CBP} 3$ is a prerequisite for post-translational modification of the carboxy-terminal of BRCA2 (aa 188-272).

CBP-mediated post-translational modification is due to $N$-linked glycosylation. The nature of this post-translational modification was investigated by use of various enzymatic digestions of the BRCA2 product (aa 1-272) pulled down by GST. Initially, we determined if this post-translational modification is due to phosphorylation. Treatment of GST pull down BRCA2 with $\lambda$ protein phosphatase (PPase) did not effect its post-translational modification (Fig. 3A, lane 4). These results suggest that such post-translational modification is not due to phosphorylation. We tested whether this posttranslational modification is due to $\mathrm{N}$ - or O-linked glycosylation. Treatment with hexosaminidase resulted in no significant change in the post-translationally modified BRCA2 (Fig. 3A, lane 3). However, digestion with $\mathrm{N}$-glycosidase $\mathrm{F}$ (PNGase F) brought the higher molecular weight, posttranslationally modified BRCA2 product down to its normal size (Fig. 3A, lane 2), suggesting that this post-translational modification is due to N-linked glycosylation. Increasing amounts of PNGase F resulted in increasing deglycosylation of BRCA2 (Fig. 3B, lanes 2-4). Pancreatic microsomal membrane (MM) failed to glycosylate (lane 5), confirming that the glycosylation of BRCA2 is mediated by CBP. BRCA2 is known to be post-translationally modified in vivo by an unknown factor(s) (6). To our knowledge, this is the first report that a transcriptional cofactor, such as CBP, is associated with post-translational, $\mathrm{N}$-linked glycosyltransferase activity.

CBP-mediated $N$-glycosylation of BRCA2 in vivo. We then verified whether this $\mathrm{N}$-glycosylation of BRCA2 occurs in vivo. MCF-7 cells were transfected with mammalian expression constructs containing CBP and BRCA2 (aa 1-272) and immunoprecipitated with CBP-specific antibody. The immunoprecipitated product was digested with PNGase F and Western

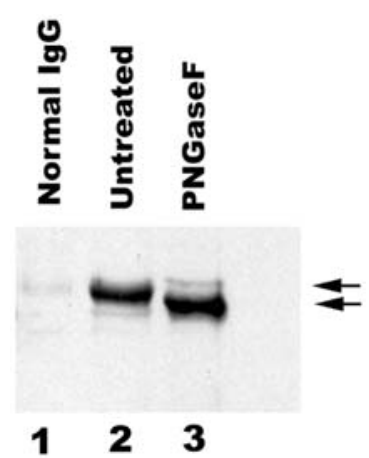

Figure 4. In vivo $\mathrm{N}$-glycosylation of BRCA2. MCF-7 cells were transfected with PCDNA/HisC BRCA2 (aa 1-272) and PCDNA-CBP. Whole cellular lysates from transfected MCF-7 cells were used for immunoprecipitation followed by PNGase F digestion. Lane 1, normal IgG; lane 2, CBP antibody; lane 3, CBP antibody and PNGase F digestion. Immunoprecipitates were separated by electrophoresis on a $10 \%$ SDS-PAGE gel, transferred to Hybond nitrocellulose paper and blotted with Xpress antibody (Invitrogen) by use of an ECL kit (Amersham). The upper arrow and the lower arrow indicate glycosylated and non-glycosylated products of BRCA2, respectively.

blotted with Xpress antibody. Untreated immunoprecipitates showed post-translationally modified BRCA2 of higher molecular weight (Fig. 4, lane 2), whereas the PNGase F-digested product had a reduced size (Fig. 4, lane 3), suggesting that BRCA2 (aa 1-272) undergoes CBP-mediated post-translational modification in vivo, as in studies performed in vitro. These results support the previous observation that BRCA2 is modified by a mechanism other than phosphorylation (6).

The tripeptide sequon Asn-Xaa-Ser/Thr is needed for $\mathrm{N}$-linked glycosylation of Asn. A search revealed no such consensus sequence in the amino-terminal region of BRCA2. However, a search for Asn sites in the amino-terminal region of BRCA2 (aa 188-272) revealed one Asn at aa 272 as a potential site for $\mathrm{N}$-linked glycosylation. To determine if this amino acid residue is indeed the site for CBP-mediated $\mathrm{N}$-linked glycosylation, deletions and point mutations of BRCA2 were made at aa 272 , and the products were tested 

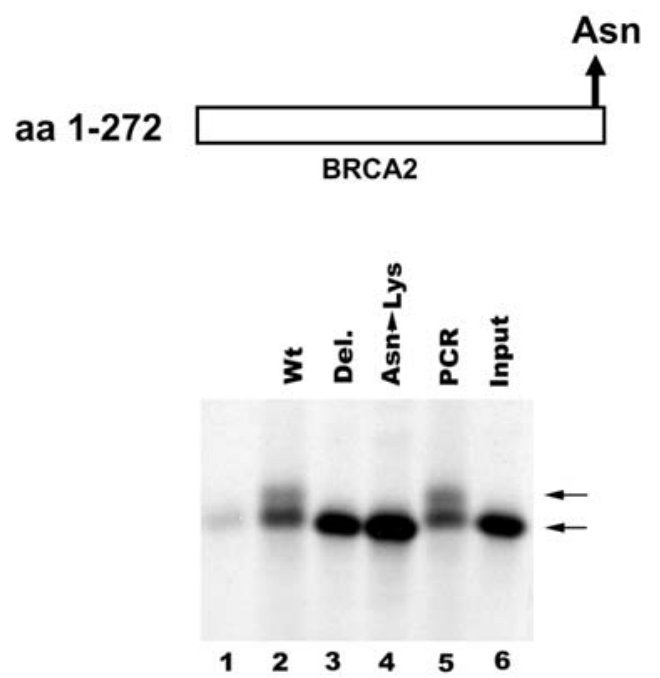

Figure 5. Identification of the CBP-mediated glycosylation site of BRCA2. Deletions and point mutations of BRCA2 were made by PCR. These constructs were linearized, translated in vitro and subjected GST-CBP3 pull down as described in Fig. 1. Lane 1, GST control; lane 2, wild-type BRCA2 (aa 1-272); lane 3, C-terminal three amino acids were deleted from BRCA2 272 [BRCA2 (aa 1-269)]; lane 4, $\operatorname{Asn}_{272}$ was mutated to Lys [BRCA2 (aa 1-272)]; lane 5, BRCA2 (aa 1-272) PCR construct to serve as an additional control for lanes 3 and 4; lane 6, input. The upper arrow and the lower arrow indicate glycosylated and non-glycosylated products of BRCA2, respectively.

for their capacity to become glycosylated. When three amino acids (aa 269-272) of the amino-terminal region of BRCA2 (which includes $\mathrm{Asn}_{272}$ ) were deleted, no glycosylation was evident (Fig. 5, lane 3). Similarly, when $\mathrm{Asn}_{272}$ of BRCA2 was mutated to Lys (Fig. 5, lane 4) and tested, no glycosylation was observed. These results allowed assignment of $\mathrm{Asn}_{272}$ as the potential glycosylation site. When the PCDNA construct was linearized with XbaI, a restriction site farther downstream of EcoRI, the in vitro translated BRCA2 was not glycosylated by CBP (data not shown). This may have been due to specific structural requirement for glycosylation of the BRCA2 protein in vitro. This is a common requirement for $\mathrm{N}$-linked glycosylation of glycoproteins in vitro by microsomal membranes.

\section{Discussion}

Typical N-linked glycosylation takes place at Asn cotranslationally when the consensus sequence Asn-Xaa-Ser/Thr is present in the proteins; this is accomplished by an oligosaccharyltransferase complex bound to the endoplasmic reticulum (7). Post-translational N-glycosylation occurs in both eukaryotic and bacterial cells (9-11). In this report, we present evidence that CBP-mediated glycosylation of BRCA2 is atypical, for there is no consensus sequence for $\mathrm{N}$-linked glycosylation. Moreover, failure of microsomal membranes to glycosylate BRCA2 indicates that the CBP-mediated gycosylation is post-translational. It is possible that the CBP HAT domain has dual enzymatic functions, which include acetylation and glycosyl transferase activity. Alternatively, CBP associates with a novel glycosyltransferase(s) that may be responsible for post-translational modification of BRCA2.
In any case, this post-translational modification represents a novel function of transcriptional co-activators such as CBP. It remains to be determined what significance CBP-mediated glycosylation of BRCA2 contributes to its biological functions, which include tumor suppression, DNA repair, the cell cycle, and apoptosis (12). Perhaps other HAT transcriptional cofactors, such as p300 and P/CAF, also function similarly to CBP and thereby regulate the activity of various interacting proteins. It is tempting to propose that CBP-mediated posttranslational $\mathrm{N}$-glycosylation may be a signal for ubiquitination and proteasomal degradation of $\mathrm{CBP} / \mathrm{p} 300$ interacting proteins. Interestingly, BRCA2 is known to be ubiquitinated and degraded by proteosomal pathway (13). It remains to be determined whether CBP-mediated post-translational N-glycosylation plays a role in the degradation of BRCA2. Since CBP/ p300 interacts with many oncoproteins, tumor suppressors and transcriptional factors, such a signal may be vital to regulate the expression of these interacting proteins which may play an important role in cell growth, differentiation and apoptosis. Thus, post-translational $\mathrm{N}$-glycosylation can have global effect on gene function, cell growth and differentiation. Microdeletions, chromosomal translocations and point mutations in CBP are linked to congenital developmental disorder, Rubinstein-Taybi syndrome (RTS), neurogenerative diseases and cancer $(14,15)$. Some of the mutations in the HAT domain of CBP are directly responsible for RTS. Since the CBP-mediated glycosyltransferase function overlaps with the HAT domain of CBP, it is possible that regulation of CBP-mediated glycosyltransferase is associated with development of RTS, neurogenerative diseases, and cancers.

\section{Acknowledgements}

This work was funded in part by GCC Distinguished Cancer Scholar award to E.S.P.R. and V.N.R., and NIH R01 CA85642, NIH R01 CA85343, DAMD 17-99-1-9060 and U56/U54 MSM/UAB/TU partnership grants to E.S.P.R. We thank K. Aysola for help with the figures.

\section{References}

1. Vo $\mathrm{N}$ and Goodman RH: CREB-binding protein and p300 in transcriptional regulation. J Biol Chem 276: 13505-13508, 2001.

2. Chan HM and La Thangue NB: p300/CBP proteins: HATs for transcriptional bridges and scaffolds. J Cell Sci 114: 2363-2373, 2001.

3. Kalkhoven E: CBP and p300: HATs for different occasions. Biochem Pharmacol 68: 1145-1155, 2004.

4. Zou JP, Hirose Y, Siddique H, Rao VN and Reddy ES: Structure and expression of variant BRCA2a lacking the transactivation domain. Oncol Rep 6: 437-440, 1999.

5. Milner J, Ponder B, Hughes-Davies L, Seltmann M and Kouzarides T: Transcriptional activation functions in BRCA2. Nature 386: 772-773, 1997.

6. Marmorstein LY, Ouchi T and Aaronson SA: The BRCA2 gene product functionally interacts with $\mathrm{p} 53$ and RAD51. Proc Natl Acad Sci USA 95: 13869-13874, 1998.

7. Helenius A and Aebi M: Roles of N-linked glycans in the endoplasmic reticulum. Annu Rev Biochem 73: 1019-1049, 2004.

8. Weerapana E and Imperiali B: Asparagine-linked protein glycosylation: from eukaryotic to prokaryotic systems. Glycobiology 16: R91-R101, 2006.

9. Bolt G, Kristensen C and Steenstrup TD: Posttranslational $\mathrm{N}$-glycosylation takes place during the normal processing of human coagulation factor VII. Glycobiology 15: 541-547, 2005. 
10. Duvet S, Op De Beeck A, Cocquerel L, Wychowski C, Cacan R and Dubuisson J: Glycosylation of the hepatitis $\mathrm{C}$ virus envelope protein E1 occurs posttranslationally in a mannosylphosphoryldolichol-deficient CHO mutant cell line. Glycobiology 12: 95-101, 2002.

11. Kowarik M, Numao S, Feldman MF, Schulz BL, Callewaert N, Kiermaier E, Catrein I and Aebi M: N-linked glycosylation of folded proteins by the bacterial oligosaccharyltransferase. Science 314: 1148-1150, 2006

12. Yoshida $\mathrm{K}$ and Miki Y: Role of BRCA1 and BRCA2 as regulators of DNA repair, transcription, and cell cycle in response to DNA damage. Cancer Sci 95: 866-871, 2004.
13. Schoenfeld AR, Apgar S, Dolios G, Wang R and Aaronson SA: BRCA2 is ubiquitinated in vivo and interacts with USP11, a deubiquitinating enzyme that exhibits prosurvival function in the cellular response to DNA damage. Mol Cell Biol 24: 7444-7455, 2004.

14. Hallam TM and Bourtchouladze R: Rubinstein-Taybi syndrome: molecular findings and therapeutic approaches to improve cognitive dysfunction. Cell Mol Life Sci 63: 1725-1735, 2006.

15. Iyer NG, Ozdag H and Caldas C: p300/CBP and cancer. Oncogene 23: 4225-4231, 2004. 